\begin{abstract}
The delivery of a beam with characteristics appropriate to the filling of the LHC proton-proton collider requires that the CERN PS Complex provides a beam whose transverse particle density exceeds, by a factor of three, the highest currently attained. This paper considers the beam dynamics operations and the associated hardware modifications which would be required to achieve this goal.

Out of a number of possible scenarios (1), the one favoured is realized in stages. Its selection is governed by the need for the shortest possible LHC filling time, especially in order to reduce the effects of persistent currents in the superconducting magnets of LHC at injection. It involves filling the Proton Synchrotron (PS) with two pulses from the Proton Synchrotron Booster (PSB) and requires an RF quadrupole (RFQ2) as a preinjector for the linac (LINAC2), an increase of the PSB extraction energy and additional RF systems, both in the PSB and PS.
\end{abstract}




\title{
The CERN PS Complex as Part of the LHC Injector Chain
}

\author{
R. Cappi, R. Garoby, S. Hancock, M. Martini, N. Rasmussen, T. Risselada, J.P. Riunaud, \\ K. Schindl, H. Schönauer, E.J.N. Wilson \\ CERN, CH-1211 Geneva 23
}

Abstract The delivery of a beam with characteristics appropriate to the filling of the LHC proton-proton collider requires that the CERN PS Complex provides a beam whose transverse particle density exceeds, by a factor of three, the highest currently attained. This paper considers the beam dynamics operations and the associated hardware modifications which would be required to achieve this goal.

Out of a number of possible scenarios [1], the one favoured is realized in stages. Its selection is governed by the need for the shortest possible LHC filling time, especially in order to reduce the effects of persistent currents in the superconducting magnets of $\mathrm{LHC}$ at injection. It involves filling the Proton Synchroton (PS) with two pulses from the Proton Synchrotron Booster (PSB) and requires an $R F$ quadrupole (RFQ2) as a preinjector for the linac (LINAC2), an increase of the PSB extraction energy and additional RF systems, both in the PSB and PS.

\section{Introduction}

Maximizing the luminosity requires the smallest crosssectional area of the two interacting beams, the highest intensity per bunch and the largest number of bunches with the shortest inter-bunch spacing. Some of the LIIC parameters for high luminosity [2] that are relevant to the PS Complex are listed in Table 1.

\begin{tabular}{|l|c|}
\hline Maximum luminosity at $\beta=0.5 \mathrm{~m}$ & $1.6510^{34} \mathrm{~cm}^{-2} \mathrm{~s}^{-1}$ \\
Number of experimental areas & 3 \\
Number of bunches & 4725 \\
Interbunch spacing & $15 \mathrm{~ns}, 4.5 \mathrm{~m}$ \\
Protons per bunch & $10^{11}$ \\
Emittances, $\dot{C}_{x, y}=\beta \gamma \sigma_{x, y}^{2} / \beta_{x, y}$ & $3.75 \mu \mathrm{m}$ \\
Longitudinal emittance at $450 \mathrm{GeV}$ & $1.0 \mathrm{eV.s}$ \\
\hline
\end{tabular}

Table 1: LHC proton-proton performance.

The roble of the PS in the injector chain for the LIIC is to deliver a $26 \mathrm{GeV} / \mathrm{c}$ beam to the SPS. In order to meet LHC requirements, the $P S$ beam must have the characteristics shown in Table 2.

Based on present high-intensity performance, which is essentially limited by the space charge induced tune spreads at injection in the PS, the PS Complex could only provide the required intensity per batch within emittances that are three times larger than those specified.

\begin{tabular}{|l|c|}
\hline Number of bunches & 140 \\
Protons per bunch & $10^{11}$ \\
Proton intensity in the PS & $1.410^{13}$ \\
Bunch spacing & $15 \mathrm{~ns}$ \\
Bunch length, $4 \sigma_{\mathrm{i}}$ & $9 \mathrm{~ns}$ \\
Long. emittance, $4 \times \sigma_{1} \sigma_{E}$ & $0.5 \mathrm{eV} . \mathrm{s}$ \\
Mean emittance, $\left(c_{x}^{*}+c_{y}^{*}\right) / 2$ & $3.0 \mu \mathrm{m}$ \\
\hline
\end{tabular}

Table 2: LHC beam characteristics at ejection from the PS.

\section{LHC filling scheme}

The existing accelerators of the PS Complex, with appropriate upgrades, can serve adequately as an injector for the LIIC. The process involved can be summarized as follows. A train of 140 bunches of $10^{11}$ protons each is formed in the PS and transferred at $26 \mathrm{GeV} / \mathrm{c}$ to the SPS. Only 135 of the total of 140 bunches can be delivered because of the rise time of the PS extraction kicker. Three such PS trains can be placed in the SPS, one after the other, yielding 405 bunches or about $410^{13}$ protons, which is presently the maximum intensity that the SPS can cope with due to beam loading. These bunches are then accelerated to $450 \mathrm{GeV} / \mathrm{c}$ and transferred to the LHC. Taking into account the rise times of the various injection and extraction kickers in the SPS and LHC, eleven SPS pulses of three PS trains each and one SPS pulse of two PS trains are necessary to fill completely one ring of the LHC.

A number of schemes have been studied to meet the LHC specification without compromising the current performance of the PS. They all involve substantial hardware modifications in the PS Complex. Among the various alternatives, the one proposed here achieves good beam characteristics for its cost and has the advantage of a reasonably short LHC filling time. The major hardware modifications are:

- an RF quadrupole, RFQ2, before LINAC2 [3],

- a new $h=1$ RF system in each of the four PSB rings,

- an increase of the PSB extraction energy from $1 \mathrm{GeV}$ to $1.4 \mathrm{GeV}$,

- a new $h=140 \mathrm{RF}$ system in the PS.

One PS train is obtained by the following steps:

1. Perform twice at $1.2 \mathrm{~s}$ intervals

1.1 delivery by LINAC2 of a $180 \mathrm{~mA}$ proton current at $50 \mathrm{MeV}$ during $7 \mu$ s using RFQ2 as a preinjector (7.5 $10^{12} \mathrm{p} /$ pulse), 
1.2 single-turn injection into the 4 PSB rings (1.75 $10^{12} \mathrm{p} / \mathrm{ring} / \mathrm{pulse}$ ),

1.3 acceleration up to $1.4 \mathrm{GeV}$ on $\mathrm{h}=1$ using the new tunable RF system (4 bunches of $1.7510^{12}$ $\mathrm{p} / \mathrm{pulse}$ ),

1.4 transfer of the 4 PSB bunches into 4 PS buckets on $h=8$ using the present PS RF system $\left(710^{12}\right.$ $p$ in half the PS circumference).

2. Acceleration up to $26 \mathrm{GeV} / \mathrm{c}$ on $h=8$ followed by debunching and rebunching on $h=140$ with the new $R F$ system.

3. Transfer to the SPS using the present fast extraction equipment.

In this scheme, the PS and SPS cycles are $3.6 \mathrm{~s}$ and $16.8 \mathrm{~s}$ long with magnetic ledges of $1.2 \mathrm{~s}$ and $7.2 \mathrm{~s}$, respectively, at injection. The corresponding LHC filling time is therefore $12 \times 16.8=201.6 \mathrm{~s}$ for one ring. The filling time is thus increased by $11 \times 2 \times 1.2=26.4 \mathrm{~s}$ compared with the fastest possible scheme involving a $2.4 \mathrm{~s}$ PS cycle.

If the LHC filling time must be reduced to the absolute minimum, e.g. in order to reduce the harmful effects of persistent currents in the superconducting magnets at injection, the proposed basic scheme can evolve towards an extended scheme at the cost of further upgrades. The fastest filling (173 s) of the LHC would then be achieved with a single batch transfer of eight PSB bunches into the PS (i.e. $3.510^{12}$ protons in two bunches per PSB ring). To reach this goal, the extended scheme will need both charge exchange $\left(\mathrm{H}^{-}\right)$injection into the PSB and an increased LINAC2 energy of $100 \mathrm{MeV}$ to reduce space charge effects. The beam will be rebunched in the PSB on $h=2$ at $1.4 \mathrm{GeV}$ using the second harmonic system which is required in the basic scheme to reduce the peak bunch current.

\section{Hardware modifications}

\section{Change of the pre-injector of LINAC2}

RFQ2, which is destined to replace the existing $750 \mathrm{keV}$ Cockcroft-Walton generator, has already demonstrated that it can deliver more than $200 \mathrm{~mA}$ of beam which is better matched to an Alvarez linac. Hence it is expected that, after its installation in 1993, the intensity and orightness of the LINAC2 beam will be substantially improved. This will be particularly attractive in connection with monoturn injection into the PSB; high-brightness beams may then be studied in the PSB and PS.

\section{Single-turn injection into the PSB}

Potentially, this injection scheme is capable of providing a circulating beam without loss of brightness. After the reinstallation of fast injection kickers, single-turn injection has been tested successfully and is available for the study of the behaviour of LHC-like beams in both the PSB and PS.

\section{Increasing the PSB output energy to $1.4 \mathrm{GeV}$}

To increase the magnetic rigidity by $20 \%$ (see section 4 ), some PSB machine components like the kickers and septa of the four rings, some dipoles and quadrupoles in the PSBto-PS transfer line, the transverse feedback amplifiers and the PS injection septum will have to be remade. Whereas the PSB main magnet operates substantially below its limits of power dissipation, magnetic saturation and voltage breakdown, the main magnet power supply needs upgrading. In addition, the water cooling plant will have to be almost completely rebuilt.

\section{New RF systems}

i. Instead of (or in addition to) the present $h=5$ system, a new PSB RF system operating on $h=1$ (i.e. covering the frequency range $0.6-1.75 \mathrm{MHz}$ ) is needed with a peak voltage of $6 \mathrm{kV}$, together with a second harmonic system providing a peak voltage of $3 \mathrm{kV}$ to flatten the longitudinal density distribution [4].

ii. While the present $9.5 \mathrm{MHz}$ cavities will be used for acceleration to $26 \mathrm{GeV} / \mathrm{c}$ on $\mathrm{h}=8$, a new PS RF system operating at $66.8 \mathrm{MHz}$ has to be installed to rebunch the beam on $h=140$ prior to extraction. The new system must be able to cope with heavy beam loading at low voltage during adiabatic capture. Its impedance must be strongly reduced during the acceleration of high intensity beams. The peak voltage required to ensure SPS beam stability at $26 \mathrm{GeV} / \mathrm{c}$ is $600 \mathrm{kV}$.

\section{Beam dynamics issues}

\section{Space charge tune shifts in the PS at injection}

At a given energy (neglecting the momentum spread of the beam and for a constant aspect ratio of the emittances), the space charge detuning is proportional to the number of particles per bunch, $N_{b}$, and inversely proportional to the bunch length, $l_{b}$, to the normalized emittances, $\epsilon_{x, y}^{*}$, and to the relativistic factor $\beta \gamma^{2}$. Assuming similar tune values in both planes, the vertical detuning may be written

$$
\Delta Q_{y} \propto \frac{N_{b}}{l_{b} \beta \gamma^{2} \epsilon_{y}^{*}}\left(1+\sqrt{\frac{\epsilon_{x}^{*}}{\epsilon_{y}^{*}}}\right)^{-1}
$$

Table 3 shows that the emittances required by the LHC are three times smaller than for the other beams listed. All other parameters being equal, the tune shift would be three times larger than at present, but, when the bunch pattern is taken into account, this factor drops to 1.5. However, the only means to compensate for this and bring the tune shift down to the present values is to increase $\beta \gamma^{2}$. This implies raising the PSB output energy from 1 to $1.4 \mathrm{GeV}$.

In order to extrapolate with confidence from present performance, a 2:1 aspect ratio for the emittances has been assumed. Taking into account transverse blow-up between the PSB and PS and in the PS itself, values of $\epsilon_{x}^{*}=3.5 \mu \mathrm{m}$ 


\begin{tabular}{|l|c|c|c|}
\hline PS beam use & LHC & $\begin{array}{c}\text { SPS } \\
\text { fixed target } \\
\text { physics }\end{array}$ & $\begin{array}{c}\text { AA/AC } \\
\text { antiproton } \\
\text { production }\end{array}$ \\
\hline Part./bunch $\left[10^{12}\right]$ & 1.75 & 1.15 & 1.5 \\
Bunch length [ns] & 197 & 55 & 90 \\
Emittance, $\epsilon_{x}^{*}[\mu \mathrm{m}]$ & 3.5 & 12.5 & 10.0 \\
Emittance, $\epsilon_{y}^{[}[\mu \mathrm{m}]$ & 1.8 & 6.3 & 5.0 \\
Kin. energy [GeV] & 1.4 & 1 & 1 \\
$\beta \gamma^{2}$ & 5.7 & 3.7 & 3.7 \\
Tune shift, $\Delta Q_{x}$ & -0.13 & -0.15 & -0.152 \\
Tune shift, $\Delta Q_{y}$ & -0.19 & -0.22 & -0.22 \\
\hline
\end{tabular}

Table 3: Characteristics of various beams at PS injection. The present high intensity beams, SPS and $A A / A C$, are compared with the expected beam for LHC.

and $\epsilon_{y}^{*}=1.8 \mu \mathrm{m}$ have been taken for the $1.410^{13}$ proton beam coming from the PSB. The final value for the aspect ratio will be chosen in the light of experience with the complete injection system.

\section{Other collective phenomena}

From the figures quoted above, it is clear that very little margin is available for transverse emittance blow-up and/or beam loss anywhere along the injector chain. Even small transverse collective effects have to be regarded as very harmful and space charge effects, in particular on the long 1.2 s magnetic ledge at PS injection, have to be carefully studied both theoretically and experimentally.

Other critical points, like transition crossing and debunching and rebunching at $26 \mathrm{GeV} / \mathrm{c}$, have also to be thoroughly investigated and optimized. Transverse coupled bunch instabilities at low energy in the PS, provoked by the resistive wall impedance, are already under control thanks to a transverse feedback system.

In the longitudinal plane, the situation is more comfortable. Longitudinal coupled bunch instabilities in the PSB will be avoided by the acceleration of a single bunch. In the PS, the total longitudinal emittance at injection is $8 \mathrm{eV} . \mathrm{s}$, while at extraction $140 \times 0.53=74 \mathrm{eV} . \mathrm{s}$ is required. This leaves quite some margin to apply controlled blow-ups to help stabilize the beam against longitudinal coupled bunch instabilities (which are also countered by longitudinal feedback) during acceleration and to avoid microwave instabilities at transition and during debunching and rebunching.

\section{Conclusions}

A scheme has been analyzed which meets the requirements of the LHC. The advantages of the scheme are a relatively modest amount of hardware modifications and the possibility of implementing these modifications gradually. The disadvantage is that little margin is left in the transverse emittance values. However, this can be offset by using three or even four PSB batches per PS pulse (increasing the LHC filling time from $200 \mathrm{~s}$ to $230 \mathrm{~s}$ or $260 \mathrm{~s}$ per ring).
Further improvements can be achieved by more significant modifications, such as installing an $\mathrm{H}^{-}$source and increasing the energy of LINAC2 to $100 \mathrm{MeV}$.

\section{References}

[1] R. Cappi, R. Garoby, S. Hancock, M. Martini, N. Rasmussen, T. Risselada, J.P. Riunaud, K. Schindl, H. Schönauer and E.J.N. Wilson, "PS Complex as Part of the LHC Injector Chain", CERN PS/91-07 (1991).

[2] The LHC Study Group, "Design Study of the Large Hadron Collider (LHC)", CERN/AC/DI/FA/90-06, (1990).

[3] E. Tanke, M. Vretenar and M. Weiss, "Performance of the CERN High-Intensity RFQ", Proc. Linear Acc. Conf., Albuquerque, N.M., Sept. 1990, p. 687.

[4] G. Gelato, L. Magnani, N. Rasmussen, K. Schindl and II. Schönauer, "Progress in Space-charge Limited Machines: Four Times the Design Intensity in the CERN Proton Synchrotron Booster", Proc. 1987 Particle Acc. Conf., Washington D.C., p. 1298. 
